\title{
ESPECIFICAÇÃO DE UM MODELO DE CRESCIMENTO E PRODUÇÃO FLORESTAL ${ }^{1}$
}

Carlos Pedro Boechat Soares ${ }^{2}$, Helio Garcia Leite ${ }^{2}$, Marcio Leles Romarco de Oliveira ${ }^{3}$ e Andrelino Carvalho ${ }^{4}$

\begin{abstract}
RESUMO - Este trabalho foi realizado com o objetivo de especificar um modelo de crescimento e produção, em que a produção volumétrica é função da área basal por hectare e da altura média de plantios de eucalipto. Para isso, foram utilizados dados de parcelas permanentes, localizadas na Bahia, com idades entre 28 e 89 meses. Após as análises, verificou-se que o modelo proposto forneceu estimativas volumétricas mais precisas do que o modelo de Clutter (1963) para povoamentos jovens, independentemente da classe de produtividade. No entanto, o modelo proposto forneceu estimativas volumétricas menos precisas nas idades técnicas de colheita (ITC), na menor e maior classes de produtividade.
\end{abstract}

Palavras-chave: Modelo de crescimento e produção, índice de local e eucalipto.

\section{SPECIFYING A MODEL OF FOREST GROWTH AND YIELD}

\begin{abstract}
The objective of this work was to specify a growth and yield model in that volumetric yield is a function of the basal area per hectare and mean height of the eucalyptus plantations. Thus, data of permanent plots located in the state of Bahia-Brazil were used, with ages ranging from 28 to 89 months. After analysis, it was verified that the proposed model provided more precise volumetric estimates than the Clutter model (1963) for young plantations, independently of the productivity class. However, the proposed model provided less precise volumetric estimates in the technical cut age (ITC) for smaller and larger productivity classes.
\end{abstract}

Key words: Growth and yield model, site index, eucalypt.

\section{INTRODUÇÃO}

Segundo Davis e Johnson (1987), a produtividade de uma floresta é definida em termos da quantidade produzida em dado período de tempo. Nesse sentido, quanto melhor a qualidade de um local, maior a produtividade da floresta, desde que esta apresente densidade populacional em grau de ocupação adequado.

As palavras "bom" e "ruim" são freqüentemente utilizadas para indicar a qualidade de um local e, simplesmente, implicam alto e baixo potencial produtivo, respectivamente (CLUTTER et al., 1983). Devido à sua subjetividade, tais expressões não são as melhores para indicar "o quanto" o local é apropriado ou não para a produção de madeira, por exemplo.

A maneira mais apropriada para verificar a qualidade de um local é através da quantificação do material produzido em um período de tempo. Porém,

\footnotetext{
${ }^{1}$ Recebido em 07.11.2002 e aceito para publicação em 10.08.2004.

${ }^{2}$ Departamento de Engenharia Florestal da UFV. E-mail: <csoares@ufv.br>e <hgleite@ufv.br>. CEP36570-000 Viçosa-MG.

${ }^{3}$ Programa de Pós-Graduação em Ciência Florestal na UFV. E- mail: <marciol @ vicosa.ufv.br>.

${ }^{4}$ COPENER LORESTAL Ltda.
} 
acontecimentos ao longo desse período de tempo, por exemplo ataques de insetos e pragas e incêndios, entre outros, podem reduzir a produção e mascarar a real capacidade produtiva do local. Diante disso, vários métodos foram desenvolvidos para determinar a qualidade de um local, entre eles os que se baseiam em características ambientais (solo, precipitação etc.), na vegetação local e na relação entre a altura e a idade das árvores. Os dois primeiros métodos apresentam alta complexidade devida às interações ambientais. O último, por sua vez, tem sido amplamente empregado, aparecendo como o mais prático e consistente indicador da qualidade do local e, conseqüentemente, da capacidade produtiva (JONES, 1969).

A altura média das árvores dominantes em uma idade específica define o chamado índice de local (CAMPOS e LEITE, 2002). De acordo com Davis e Johnson (1987), o índice de local não é perfeito, porém, como as árvores dominantes estão crescendo livres de competição no estrato superior, elas estão fortemente correlacionadas com o volume do povoamento e não são afetadas, dentro de certos limites, pela densidade populacional, apresentando vantagens em comparação com os outros métodos. Além disso, assume-se que os indivíduos dominantes conseguem captar os recursos ambientais a eles oferecidos, expressando isso em seu desenvolvimento, isto é, em suas alturas.

A utilização do índice de local permite ao manejador avaliar diferentes alternativas de manejo quando associadas a um modelo de crescimento e produção. Um exemplo clássico de modelo, em nível de povoamento, que permite simular diversas opções de manejo é o de Clutter (1963), cujas relações funcionais são:

$$
\begin{aligned}
& \ln V_{2}=\beta_{0}+\beta_{1} \cdot\left(1 / I_{2}\right)+\beta_{2} \cdot S+\beta_{3} \cdot \ln B_{2}+\varepsilon \\
& \ln B_{2}=\ln B_{1} \cdot\left(I_{I} / I_{2}\right)+\beta_{4} \cdot\left(1-I_{I} / I_{2}\right)+\beta_{5} \cdot\left(1-I_{I} / I_{2}\right) \cdot S+\varepsilon
\end{aligned}
$$

em que $\ln =$ logaritmo neperiano; $V_{2}=$ volume futuro por hectare; $I_{2}=$ idade futura; $I_{1}=$ idade presente; $B_{2}$ = área basal futura por hectare; $B_{1}=$ área basal presente por hectare; $S=$ índice de local; $\beta_{0} \ldots \beta_{5}=$ parâmetros do modelos; e $\varepsilon=$ erro aleatório.

Do ponto de vista teórico, esse modelo está corretamente especificado, pela inclusão de variáveis do povoamento relacionadas à produção volumétrica (idade, índice de local e área basal). Observa-se, contudo, no modelo anterior, que o índice de local é uma variável independente na equação que projeta a produção volumétrica. Conceitualmente, isso conflita com o mencionado por Spurr (1952), uma vez que, segundo esse autor, a produção volumétrica de uma floresta é função da área basal por hectare e da altura média das árvores $(\bar{H})$ e não da altura média das árvores dominantes, em que se fundamenta o índice de local.

Como complemento, Leary (1988) mostrou, através de uma matriz de variáveis relacionadas à produção volumétrica, que a altura média das árvores de uma floresta é função da idade e da capacidade produtiva do local. Esse comportamento da variável altura média permite inferir sobre a possibilidade de especificação de um modelo de crescimento e produção, composto por um sistema de equações, em que a produção volumétrica de uma floresta seja função de variáveis como idade, área basal por hectare e altura média das árvores, tendo o índice de local como uma variável diferenciadora da produção.

Diante desses argumentos, este trabalho teve por objetivo especificar um modelo de crescimento e produção, composto por um sistema de equações, tendo como referência o modelo de Clutter (1963).

\section{MATERIAL E MÉTODOS}

Os dados utilizados neste estudo foram provenientes de 160 parcelas permanentes de plantios de Eucalyptus grandis W. Hill ex Maiden x E. urophylla S. T. Blake, localizados no município de Entre Rios, Estado da Bahia, pertencentes à empresa COPENER Florestal Ltda. Esses dados compreenderam plantios com idades entre 28 e 89 meses, área basal entre 2,11 e $35,89 \mathrm{~m}^{2} / \mathrm{ha}$, volume total com casca variando de 18,86 a $489,18 \mathrm{~m}^{3} /$ ha e índice de local entre 12,48 e $36,06 \mathrm{~m}$.

Para a classificação da capacidade produtiva dos plantios, através do índice de local, utilizou-se a seguinte equação:

$$
\begin{aligned}
& \ln H d=3,625923-26,3768 .(1 / I) ; \text { Idade-índice de } 60 \text { meses } \\
& \mathrm{R}^{2}=52,64 \% \\
& \text { em que: } \\
& \text { ln = logaritmo neperiano; } \\
& H d=\text { altura média das árvores dominantes, em m; e } \\
& I=\text { idade, em meses. }
\end{aligned}
$$

Inicialmente, utilizando o método dos Mínimos Quadrados em Dois Estágios (MQ2E), foi ajustado o modelo de Clutter, na sua forma original, conforme 
descrito anteriormente. A escolha desse modelo baseouse no seu amplo emprego no setor florestal e, em especial, por ser um modelo compatível e consistente.

A precisão das equações foi verificada analisandose os coeficientes de determinação $\left(R^{2}\right)$ e os coeficientes de variação $(\mathrm{CV})$, na unidade original $\left(\mathrm{m}^{3} / \mathrm{ha}\right)$, e a exatidão das estimativas, através do comportamento dos gráficos dos valores estimados em relação aos valores observados.

Em seguida, foram realizadas e testadas modificações no modelo, adequando-o ao uso coerente das variáveis altura média das árvores do povoamento (média aritmética $-\bar{H}$ ) e do índice de local, mantendo a propriedade de compatibilidade e consistência. Após as modificações, o modelo proposto foi ajustado utilizando-se a mesma base de dados e comparado com o ajuste do modelo original de Clutter.

\section{RESULTADOS E DISCUSSÃO}

Ao ajustar o modelo de Clutter (1963) na sua forma original, obteve-se:

$$
\begin{aligned}
\ln V_{2}= & 1,679631-21,57754 \cdot\left(1 / I_{2}\right)+0,019446 \cdot S+1,169825 \cdot \ln B_{2} \\
& \mathrm{R}^{2}=98,97 \% ; \mathrm{CV}= \pm 9,67 \% \\
\ln B_{2}= & \ln B_{1} \cdot\left(I_{I} / I_{2}\right)+2,807449 \cdot\left(1-I_{I} / I_{2}\right)+0,026929 \cdot(1- \\
& \left.I_{I} / I_{2}\right) \cdot S \\
& \mathrm{R}^{2}=94,82 \% ; \mathrm{CV}= \pm 7,18 \%
\end{aligned}
$$

Pela análise da Figura 1, relacionando os volumes estimados em função dos volumes observados, verificouse a exatidão das estimativas da produção volumétrica, haja vista a tendência apresentada $\left(45^{\circ}\right.$, passando pela origem). No entanto, cabe ressaltar a leve tendência de subestimação em plantios com maior produção volumétrica (acima de $350 \mathrm{~m}^{3} / \mathrm{ha}$ ).
Utilizando três classes de produtividade $(S=15$, $\mathrm{S}=23$ e $\mathrm{S}=31$ ), projetaram-se as áreas basais e os volumes com casca por hectare até a idade de 90 meses, bem como foram calculados os respectivos incrementos médios mensais em volume (IMM), visando obter as idades técnicas de colheita (ITC) para as classes de produtividade consideradas (Quadro 1). As áreas basais iniciais, aos 28 meses, nas três classes de produtividade, foram obtidas pela média aritmética das áreas basais nessa idade.

Verifica-se, no Quadro 1, que as idades técnicas de colheita nas classes de local 15, 23 e 31 foram, respectivamente, iguais a 76, 60 e 58 meses, com apenas dois meses de diferença entre as ITC, nas classes 23 e 31 .

Considerando a forma estrutural do modelo de Clutter (1963) e as tendências apresentadas no trabalho de Leary (1988), foram feitas modificações no modelo original, definindo a seguinte forma estrutural para o modelo proposto:

$$
\begin{aligned}
& \ln V_{2}=\beta_{0}+\beta_{1} \cdot\left(1 / I_{2}\right)+\beta_{2} \cdot \operatorname{Ln} B_{2}+\beta_{3} \cdot \ln \bar{H}_{2}+\varepsilon \\
& \ln B_{2}=\ln B_{1} \cdot\left(I_{1} / I_{2}\right)+\beta_{4} \cdot\left(1-I_{1} / I_{2}\right)+\beta_{5} \cdot\left(1-I_{1} / I_{2}\right) \cdot S+\varepsilon \\
& \ln \bar{H}_{2}=\ln \bar{H}_{1} \cdot\left(I_{1} / I_{2}\right)+\beta_{6} \cdot\left(1-I_{1} / I_{2}\right)+\beta_{7} \cdot\left(1-I_{1} / I_{2}\right) \cdot S+\varepsilon
\end{aligned}
$$

em que $V_{2}=$ volume total com casca futuro, $\mathrm{em} \mathrm{m}^{3} / \mathrm{ha}$; $I_{2}=$ idade futura, em meses; $I_{1}=$ idade presente, em meses; $B_{2}=$ área basal futura, $\mathrm{em} \mathrm{m}{ }^{2} / \mathrm{ha} ; B_{1}=$ área basal presente, em m²/ha; $\bar{H}_{2}=$ altura média futura, em metros; $\bar{H}_{1}=$ altura média presente, em metros; $S=$ índice de local, em metros; $\beta_{0} \ldots \beta_{7}=$ parâmetros do modelos; e $\varepsilon=$ erro aleatório. Dessa forma, quando $\mathrm{I}_{1}$ for igual a $\mathrm{I}_{2}, \bar{H}_{2}=\bar{H}_{1}$ e $\mathrm{B}_{2}=\mathrm{B}_{1}$.

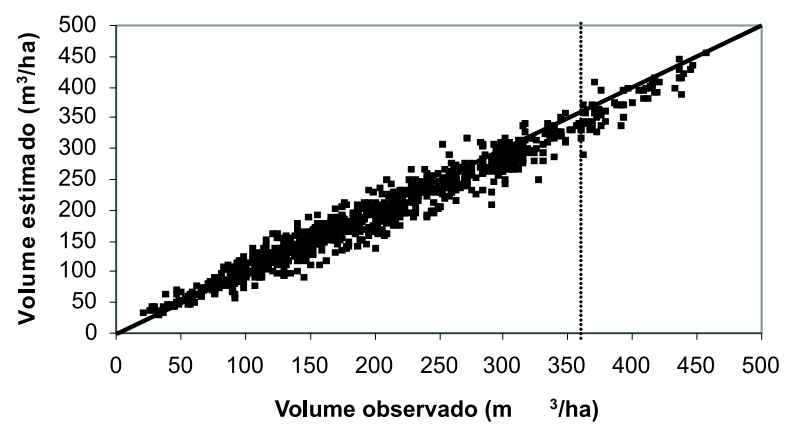

Figura 1 - Volumes observados e estimados por hectare pelo modelo de Clutter (1963). Figure 1 - Observed and estimated volumes per hectare obtained by the Clutter model (1963). 
Quadro 1 - Estimativas de área basal por hectare (B), volume por hectare (Vol) e incremento médio mensal em volume (IMM), nas três classes de índices de locais (S), obtidas pelo modelo de Clutter (1963)

Table 1 - Estimates of basal area per hectare (B), volume per hectare (Vol) and mean monthly increment (IMM) in volume, for three site index classes (S), obtained by Clutter model (1963)

\begin{tabular}{|c|c|c|c|c|c|c|c|c|c|}
\hline \multirow[b]{2}{*}{ Idade } & \multicolumn{3}{|c|}{$\begin{array}{l}\text { Classe de Produtividade III } \\
(\mathrm{S}=15)\end{array}$} & \multicolumn{3}{|c|}{$\begin{array}{l}\text { Classe de Produtividade II } \\
(\mathrm{S}=23)\end{array}$} & \multicolumn{3}{|c|}{$\begin{array}{l}\text { Classe de Produtividade I } \\
\qquad(\mathrm{S}=31)\end{array}$} \\
\hline & $\begin{array}{c}\text { B } \\
\left(\mathrm{m}^{2} / \mathrm{ha}\right) \\
\end{array}$ & $\begin{array}{c}\text { Vol. } \\
\left(\mathrm{m}^{3} / \mathrm{ha}\right)\end{array}$ & $\begin{array}{c}\text { IMM } \\
\left(\mathrm{m}^{3} / \text { ha.ano }\right) \\
\end{array}$ & $\begin{array}{c}\text { B } \\
\left(\mathrm{m}^{2} / \mathrm{ha}\right) \\
\end{array}$ & $\begin{array}{c}\text { Vol. } \\
\left(\mathrm{m}^{3} / \mathrm{ha}\right)\end{array}$ & $\begin{array}{c}\text { IMM } \\
\left(\mathrm{m}^{3} / \text { ha.ano }\right) \\
\end{array}$ & $\begin{array}{c}\text { B } \\
\left(\mathrm{m}^{2} / \mathrm{ha}\right) \\
\end{array}$ & $\begin{array}{c}\text { Vol. } \\
\left(\mathrm{m}^{3} / \mathrm{ha}\right)\end{array}$ & $\begin{array}{c}\text { IMM } \\
\left(\mathrm{m}^{3} / \text { ha.ano }\right) \\
\end{array}$ \\
\hline 28 & 4,6471 & 20,0420 & 0,7158 & 9,3941 & 53,3442 & 1,9051 & 12,4419 & 86,5772 & 3,0920 \\
\hline 30 & 5,1962 & 24,0431 & 0,8014 & 10,1675 & 61,6018 & 2,0534 & 13,4075 & 99,4695 & 3,3156 \\
\hline 32 & 5,7296 & 28,1941 & 0,8811 & 10,8963 & 69,8694 & 2,1834 & 14,3137 & 112,3157 & 3,5099 \\
\hline 34 & 6,2455 & 32,4480 & 0,9544 & 11,5826 & 78,0810 & 2,2965 & 15,1640 & 125,0216 & 3,6771 \\
\hline 36 & 6,7430 & 36,7653 & 1,0213 & 12,2289 & 86,1871 & 2,3941 & 15,9621 & 137,5175 & 3,8199 \\
\hline 38 & 7,2216 & 41,1127 & 1,0819 & 12,8376 & 94,1507 & 2,4776 & 16,7118 & 149,7531 & 3,9409 \\
\hline 40 & 7,6813 & 45,4633 & 1,1366 & 13,4113 & 101,9451 & 2,5486 & 17,4165 & 161,6932 & 4,0423 \\
\hline 42 & 8,1224 & 49,7950 & 1,1856 & 13,9525 & 109,5519 & 2,6084 & 18,0798 & 173,3145 & 4,1265 \\
\hline 44 & 8,5453 & 54,0900 & 1,2293 & 14,4634 & 116,9585 & 2,6581 & 18,7046 & 184,6026 & 4,1955 \\
\hline 46 & 8,9507 & 58,3342 & 1,2681 & 14,9462 & 124,1576 & 2,6991 & 19,2939 & 195,5499 & 4,2511 \\
\hline 48 & 9,3392 & 62,5168 & 1,3024 & 15,4029 & 131,1453 & 2,7322 & 19,8504 & 206,1544 & 4,2949 \\
\hline 50 & 9,7115 & 66,6290 & 1,3326 & 15,8353 & 137,9209 & 2,7584 & 20,3766 & 216,4177 & 4,3284 \\
\hline 52 & 10,0682 & 70,6647 & 1,3589 & 16,2453 & 144,4856 & 2,7786 & 20,8746 & 226,3445 & 4,3528 \\
\hline 54 & 10,4103 & 74,6190 & 1,3818 & 16,6344 & 150,8423 & 2,7934 & 21,3466 & 235,9415 & 4,3693 \\
\hline 56 & 10,7383 & 78,4887 & 1,4016 & 17,0040 & 156,9951 & 2,8035 & 21,7945 & 245,2169 & 4,3789 \\
\hline 58 & 11,0529 & 82,2718 & 1,4185 & 17,3555 & 162,9489 & 2,8095 & $\underline{22,2199}$ & 254,1802 & 4,3824 \\
\hline 60 & 11,3549 & 85,9670 & 1,4328 & $\underline{17,6901}$ & 168,7094 & 2,8118 & & $\overline{262,8413}$ & 4,3807 \\
\hline 62 & 11,6449 & 89,5739 & 1,4447 & 18,0090 & 174,2825 & 2,8110 & 23,0095 & 271,2106 & 4,3744 \\
\hline 64 & 11,9235 & 93,0927 & 14546 & 18,3131 & 179,6743 & 2,8074 & 23,3765 & 279,2986 & 4,3640 \\
\hline 66 & 12,1913 & 96,5240 & 1,4625 & 18,6036 & 184,8912 & 2,8014 & 23,7266 & 287,1161 & 4,3502 \\
\hline 68 & 12,4488 & 99,8690 & 14687 & 18,8811 & 189,9395 & 2,7932 & 24,0608 & 294,6735 & 4,3334 \\
\hline 70 & 12,6965 & 103,1289 & 1,4733 & 19,1465 & 194,8255 & 2,7832 & 24,3803 & 301,9812 & 4,3140 \\
\hline 72 & 12,9351 & 106,3054 & 1,4765 & 19,4007 & 199,5555 & 2,7716 & 24,6859 & 309,0491 & 4,2923 \\
\hline 74 & 13,1648 & 109,4001 & 1,4784 & 19,6442 & 204,1354 & 2,7586 & 24,9785 & 315,8873 & 4,2687 \\
\hline 76 & $\underline{13,3862}$ & 112,4151 & 1,4791 & 19,8777 & 208,5711 & 2,7 & 25,2590 & 322,5050 & 4,2435 \\
\hline 78 & 13,5998 & 115,3522 & 1,4789 & 20,1018 & 212,8685 & & 25,5279 & & 4,2168 \\
\hline 80 & 13,8058 & 118,2135 & 1,4777 & 20,3170 & 217,0330 & 2,7129 & 25,7861 & 335,1155 & 4,1889 \\
\hline 82 & 14,0046 & 121,0010 & 1,4756 & 20,5239 & 221,0699 & 2,6960 & 26,0341 & 341,1254 & 4,1601 \\
\hline 84 & 14,1966 & 123,7169 & 1,4728 & 20,7229 & 224,9844 & 2,6784 & 26,2724 & 346,9493 & 4,1303 \\
\hline 86 & 14,3822 & 126,3633 & 1,4693 & 20,9144 & 228,7813 & 2,6602 & 26,5018 & 352,5950 & 4,0999 \\
\hline 88 & 14,5616 & 128,9421 & 1,4653 & 21,0988 & 232,4654 & 2,6417 & 26,7226 & 358,0696 & 4,0690 \\
\hline 90 & 14,7351 & 131,4555 & 1,4606 & 21,2766 & 236,0412 & 2,6227 & 26,9353 & 363,3804 & 4,0376 \\
\hline
\end{tabular}

Utilizando a mesma base de dados, ajustou-se o modelo proposto empregando o método dos Mínimos Quadrados em Dois Estágios (MQ2E), resultando em:

$$
\begin{aligned}
\ln V_{2}=- & 0,253687-4,748820 \cdot\left(1 / I_{2}\right)+1,032913 \cdot \ln B_{2}+ \\
& 0,837185 \cdot \ln \bar{H}_{2} \\
& \mathrm{R}^{2}=99,69 \% ; \mathrm{C} \cdot \mathrm{V}= \pm 1,15 \% \\
\ln B_{2}= & \ln B_{1} \cdot\left(I_{1} / I_{2}\right)+2,807449 \cdot\left(1-I_{1} / I_{2}\right)+0,026929 \cdot\left(1-I_{1} / I_{2}\right) \cdot S \\
& \mathrm{R}^{2}=94,82 \% ; \mathrm{C} \cdot \mathrm{V}= \pm 7,18 \% \\
\ln \bar{H}_{2}= & \ln \bar{H}_{1} \cdot\left(I_{1} / I_{2}\right)+3,062917 \cdot\left(1-I_{1} / I_{2}\right)+0,014519 \cdot\left(1-I_{1} / I_{2}\right) \cdot S \\
& \mathrm{R}^{2}=93,49 \% ; \mathrm{C} \cdot \mathrm{V}= \pm 4,27 \%
\end{aligned}
$$

em que todos os coeficientes foram significativos em nível de $1 \%$ de significância, pelo teste "t" (Student).

Pelo valor do coeficiente de determinação $\left(\mathrm{R}^{2}\right)$ da equação que projeta a produção volumétrica, verificou- se que o modelo proposto apresentou-se mais preciso do que o modelo original de Clutter (1963). No entanto, verificou-se novamente uma tendência de subestimação dos volumes nos povoamentos acima de $300 \mathrm{~m}^{3} / \mathrm{ha}$ (Figura 2).

Considerando as idades técnicas de colheita (ITC) de 78, 60 e 54 meses (Quadro 2), nas quais as projeções volumétricas são menores que $300 \mathrm{~m}^{3} /$ ha, notou-se que a tendência de subestimação não invalida o uso do modelo proposto, uma vez que os plantios estariam sendo colhidos antes da idade em que se verifica tal tendenciosidade.

Comparando os dois modelos em termos de idade técnica de colheita (ITC), observa-se, na Figura 3, que o modelo proposto propicia um aumento da ITC nos locais com menores produtividades e diminuição da 
ITC em plantios de maiores produtividades. Na prática, como a colheita de plantios leva alguns meses para ser efetuada, a diferença entre as idades técnicas de colheita obtidas pelo modelo de Clutter (1963) e pelo modelo proposto pode ser considerada nãosignificativa.

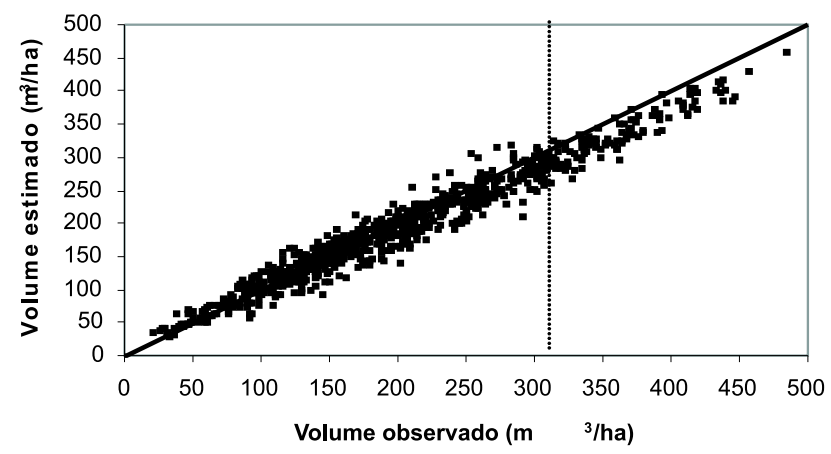

Figura 2 - Volumes observados e estimados por hectare, obtidos pelo modelo proposto.

Figure 2 - Observed and estimated volumes per hectare obtained by the proposed model.

Quadro 2 - Estimativas de área basal por hectare (B), altura média (Ht), volume por hectare (V) e incremento médio mensal em volume (IMM), nas três classes de produtividade (S), obtidas pelo modelo proposto

Table 2 - Estimates of basal area per hectare $(B)$, mean height $(\mathrm{Ht})$, volume per hectare (Vol) and mean monthly increment $(I M M)$ in volume, for three site indexes classes $(S)$, obtained by the proposed model

\begin{tabular}{|c|c|c|c|c|c|c|c|c|c|c|c|c|}
\hline \multirow[b]{2}{*}{ Idade } & \multicolumn{4}{|c|}{$\begin{array}{l}\text { Classe de Produtividade III } \\
\qquad(\mathrm{S}=15)\end{array}$} & \multicolumn{4}{|c|}{$\begin{array}{l}\text { Classe de Produtividade II } \\
\qquad(\mathrm{S}=23)\end{array}$} & \multicolumn{4}{|c|}{$\begin{array}{l}\text { Classe de Produtividade I } \\
\qquad(\mathrm{S}=31)\end{array}$} \\
\hline & $\begin{array}{c}\text { B } \\
\left(\mathrm{m}^{2} / \mathrm{ha}\right)\end{array}$ & $\begin{array}{l}\mathrm{Ht} \\
(\mathrm{m})\end{array}$ & $\begin{array}{c}\text { Vol. } \\
\left(\mathrm{m}^{3} / \mathrm{ha}\right)\end{array}$ & $\begin{array}{c}\text { IMM } \\
\left(\mathrm{m}^{3} / \text { ha.ano) }\right.\end{array}$ & $\begin{array}{c}\text { B } \\
\left(\mathrm{m}^{2} / \mathrm{ha}\right)\end{array}$ & $\begin{array}{l}\mathrm{Ht} \\
(\mathrm{m})\end{array}$ & $\begin{array}{c}\text { Vol. } \\
\left(\mathrm{m}^{3} / \mathrm{ha}\right)\end{array}$ & $\begin{array}{c}\text { IMM } \\
\left(\mathrm{m}^{3} / \text { ha.ano }\right)\end{array}$ & $\begin{array}{c}\text { B } \\
\left(\mathrm{m}^{2} / \mathrm{ha}\right)\end{array}$ & $\begin{array}{l}\mathrm{Ht} \\
(\mathrm{m})\end{array}$ & $\begin{array}{c}\text { Vol. } \\
\left(\mathrm{m}^{3} / \mathrm{ha}\right)\end{array}$ & $\begin{array}{c}\text { IMM } \\
\left(\mathrm{m}^{3} / \text { ha.ano }\right)\end{array}$ \\
\hline 28 & 4,6471 & 9,31 & 20,7299 & 0,7404 & 9,3941 & 12,49 & 54,8281 & 1,9581 & 12,4419 & 15,88 & 89,6124 & 3,2004 \\
\hline 30 & 5,1962 & 9,99 & 24,9482 & 0,8316 & 10,1675 & 13,24 & 63,1747 & 2,1058 & 13,4075 & 16,69 & 102,0802 & 3,4027 \\
\hline 32 & 5,7296 & 10,62 & 29,3377 & 0,9168 & 10,8963 & 13,93 & 71,5139 & 2,2348 & 14,3137 & 17,43 & 114,4046 & 3,5751 \\
\hline 34 & 6,2455 & 11,21 & 33,8480 & 0,9955 & 11,5826 & 14,57 & 79,7816 & 2,3465 & 15,1640 & 18,12 & 126,5090 & 3,7209 \\
\hline 36 & 6,7430 & 11,76 & 38,4360 & 1,0677 & 12,2289 & 15,16 & 87,9297 & 2,4425 & 15,9621 & 18,75 & 138,3396 & 3,8428 \\
\hline 38 & 7,2216 & 12,27 & 43,0657 & 1,1333 & 12,8376 & 15,71 & 95,9231 & 2,5243 & 16,7118 & 19,33 & 149,8596 & 3,9437 \\
\hline 40 & 7,6813 & 12,76 & 47,7075 & 1,1927 & 13,4113 & 16,22 & 103,7366 & 2,5934 & 17,4165 & 19,87 & 161,0456 & 4,0261 \\
\hline 42 & 8,1224 & 13,21 & 52,3369 & 1,2461 & 13,9525 & 16,70 & 111,3530 & 2,6513 & 18,0798 & 20,37 & 171,8841 & 4,0925 \\
\hline 44 & 8,5453 & 13,64 & 56,9341 & 1,2940 & 14,4634 & 17,15 & 118,7612 & 2,6991 & 18,7046 & 20,84 & 182,3690 & 4,1447 \\
\hline 46 & 8,9507 & 14,04 & 61,4833 & 1,3366 & 14,9462 & 17,57 & 125,9548 & 2,7381 & 19,2939 & 21,28 & 192,4997 & 4,1848 \\
\hline 48 & 9,3392 & 14,42 & 65,9721 & 1,3744 & 15,4029 & 17,96 & 132,9311 & 2,7694 & 19,8504 & 21,68 & 202,2799 & 4,2142 \\
\hline 50 & 9,7115 & 14,78 & 70,3907 & 1,4078 & 15,8353 & 18,33 & 139,6901 & 2,7938 & 20,3766 & 22,07 & 211,7159 & 4,2343 \\
\hline 52 & 10,0682 & 15,11 & 74,7316 & 1,4371 & 16,2453 & 18,68 & 146,2338 & 2,8122 & 20,8746 & 22,43 & 220,8162 & 4,2465 \\
\hline 54 & 10,4103 & 15,43 & 78,9892 & 1,4628 & 16,6344 & 19,00 & 152,5658 & 2,8253 & $\underline{21,3466}$ & $\underline{22,76}$ & $\underline{229,5909}$ & $\underline{4,2517}$ \\
\hline 56 & 10,7383 & 15,74 & 83,1597 & 1,4850 & 17,0040 & 19,31 & 158,6907 & 2,8338 & $\overline{21,7945}$ & $\overline{23,08}$ & $\overline{238,0508}$ & $\overline{4,2509}$ \\
\hline 58 & 11,0529 & 16,02 & 87,2403 & 1,5041 & 17,3555 & 19,61 & 164,6141 & 2,8382 & 22,2199 & 23,38 & 246,2072 & 4,2450 \\
\hline 60 & 11,3549 & 16,30 & 91,2292 & 1,5205 & 17,6901 & $\underline{19,88}$ & $\mathbf{1 7 0 , 3 4 1 8}$ & 2,8390 & 22,6244 & 23,66 & 254,0717 & 4,2345 \\
\hline 62 & 11,6449 & 16,56 & 95,1258 & 1,5343 & $\overline{18,0090}$ & $\overline{20,15}$ & $\overline{175,8804}$ & $\overline{2,8368}$ & 23,0095 & 23,93 & 261,6562 & 4,2203 \\
\hline 64 & 11,9235 & 16,80 & 98,9298 & 1,5458 & 18,3131 & 20,40 & 181,2362 & 2,8318 & 23,3765 & 24,18 & 268,9721 & 4,2027 \\
\hline 66 & 12,1913 & 17,04 & 102,6418 & 1,5552 & 18,6036 & 20,63 & 186,4159 & 2,8245 & 23,7266 & 24,43 & 276,0309 & 4,1823 \\
\hline 68 & 12,4488 & 17,26 & 106,2626 & 1,5627 & 18,8811 & 20,86 & 191,4261 & 2,8151 & 24,0608 & 24,65 & 282,8436 & 4,1595 \\
\hline 70 & 12,6965 & 17,48 & 109,7933 & 1,5685 & 19,1465 & 21,07 & 196,2732 & 2,8039 & 24,3803 & 24,87 & 289,4209 & 4,1346 \\
\hline 72 & 12,9351 & 17,68 & 113,2356 & 1,5727 & 19,4007 & 21,28 & 200,9637 & 2,7912 & 24,6859 & 25,08 & 295,7732 & 4,1080 \\
\hline 74 & 13,1648 & 17,88 & 116,5911 & 1,5756 & 19,6442 & 21,47 & 205,5038 & 2,7771 & 24,9785 & 25,28 & 301,9104 & 4,0799 \\
\hline 76 & 13,3862 & 18,07 & 119,8616 & 1,5771 & 19,8777 & 21,66 & 209,8995 & 2,7618 & 25,2590 & 25,47 & 307,8420 & 4,0506 \\
\hline 78 & $\underline{13,5998}$ & 18,25 & $\underline{123,0492}$ & $\underline{\mathbf{1}, \mathbf{5 7 7 6}}$ & 20,1018 & 21,84 & 214,1567 & 2,7456 & 25,5279 & 25,65 & 313,5771 & 4,0202 \\
\hline 80 & 13,8058 & 18,42 & 126,1559 & 1,5769 & 20,3170 & 22,01 & 218,2810 & 2,7285 & 25,7861 & 25,82 & 319,1244 & 3,9891 \\
\hline 82 & 14,0046 & 18,59 & 129,1838 & 1,5754 & 20,5239 & 22,18 & 222,2778 & 2,7107 & 26,0341 & 25,99 & 324,4922 & 3,9572 \\
\hline 84 & 14,1966 & 18,74 & 132,1351 & 1,5730 & 20,7229 & 22,33 & 226,1522 & 2,6923 & 26,2724 & 26,14 & 329,6882 & 3,9249 \\
\hline 86 & 14,3822 & 18,90 & 135,0118 & 1,5699 & 20,9144 & 22,49 & 229,9094 & 2,6734 & 26,5018 & 26,30 & 334,7201 & 3,8921 \\
\hline 88 & 14,5616 & 19,05 & 137,8162 & 1,5661 & 21,0988 & 22,63 & 233,5539 & 2,6540 & 26,7226 & 26,44 & 339,5948 & 3,8590 \\
\hline 90 & 14,7351 & 19,19 & 140,5504 & 1,5617 & 21,2766 & 22,77 & 237,0905 & 2,6343 & 26,9353 & 26,58 & 344,3192 & 3,8258 \\
\hline
\end{tabular}




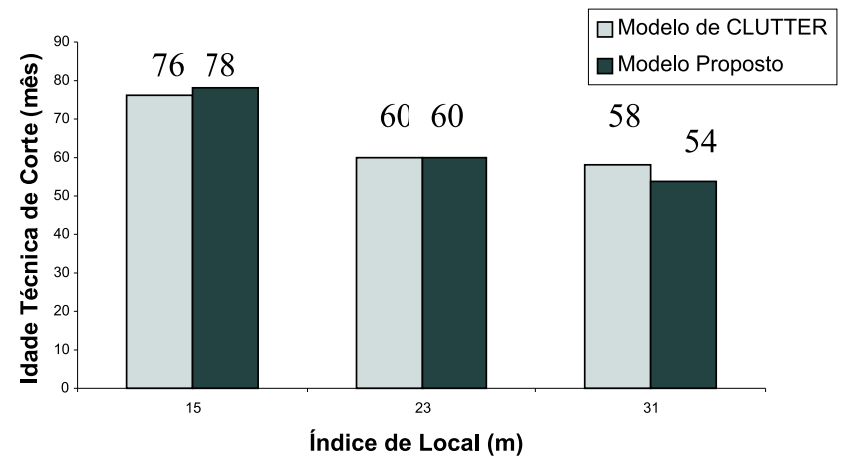

Figura 3 - Idades técnicas de corte (ITC's) pelo modelo de Clutter (1963) e pelo modelo proposto, nas três classes de produtividade $(\mathrm{S}=15, \mathrm{~S}=23$ e $\mathrm{S}=31)$

Figure 3 -Technical cut ages (ITC) for the proposed and CLUTTER model (1963) considering three site index classes ( $S=15$, $S=23$ e $S=31$ ).

Analisando os volumes nas idades técnicas de colheita (ITC), apresentados no Quadro 3, verificouse que o modelo de Clutter (1963) projetou volumes menores nas classes iguais a 15 e 23 . Na classe 31 , observou-se o contrário.

Para verificar a precisão das estimativas volumétricas obtidas pelos modelos ajustados em relação aos volumes observados, foram calculadas as médias dos desvios

Quadro 3 - Diferenças entre os volumes projetados pelo modelo de Clutter (C) e pelo modelo proposto (P), nas idades técnicas de colheita (ITC)

Table 3 - Differences between projected volumes by CLUTTER $(C)$ and proposed $(P)$ models in the technical cut age (ITC)

\begin{tabular}{lccccc}
\hline & $\begin{array}{c}\text { ITC } \\
(\text { meses })\end{array}$ & \multicolumn{2}{c}{$\begin{array}{c}\text { Volumes } \\
\left(\mathrm{m}^{3} / \mathrm{ha}\right)\end{array}$} & & $\begin{array}{c}\text { Diferença } \\
(\%)\end{array}$ \\
\cline { 3 - 4 } $\mathrm{S}(\mathrm{m})$ & & Clutter $(\mathrm{C})$ & Proposto $(\mathrm{P})$ & & $\mathrm{P}-\mathrm{C}$ \\
\hline 15 & 76 & 112,4151 & 119,8616 & \\
15 & 78 & 115,3522 & 123,0492 & & 6,67 \\
23 & 60 & 168,7094 & 170,3418 & & 0,97 \\
31 & 54 & 235,9415 & 229,5909 & & $-2,69$ \\
31 & 58 & 254,1802 & 246,2072 & & $-3,13$ \\
\hline
\end{tabular}

\section{CONCLUSÕES}

Com base nos resultados deste estudo, concluiuse que:

a) O modelo proposto apresentou-se mais preciso do que o de Clutter (1963), em idades menores do que as idades técnicas de colheita (ITC), independentemente da classe de produtividade.

R. Árvore, Viçosa-MG, v.28, n.6, p.831-837, 2004 porcentuais por classe de idade, nas três classes de produtividades (Quadro 4). O modelo proposto apresentou-se mais preciso em plantios até 77 meses, na classe igual a 15, e até 47 meses na classe 31 . Nas idades técnicas de colheita (ITC) nas duas classes, as estimativas foram menos precisas (valores sublinhados). Apenas na classe igual a 23 o modelo proposto propiciou estimativas mais precisas na ITC.

Quadro 4 - Médias dos desvios porcentuais por classe de idade, nas três classes de produtividade

Table 4-Average of the percent deviations for ages and productivity classes

\begin{tabular}{lcccccc}
\hline & \multicolumn{2}{c}{$\mathrm{S}=15$} & \multicolumn{2}{c}{$\mathrm{S}=23$} & \multicolumn{2}{c}{$\mathrm{S}=31$} \\
\cline { 2 - 7 } $\begin{array}{l}\text { Idade } \\
\text { (meses) }\end{array}$ & Clutter & Proposto & Clutter & Proposto & Clutter & Proposto \\
\hline $28-37$ & 29,42 & 28,52 & 3,11 & 4,22 & 5,88 & 5,45 \\
$38-47$ & 0,40 & $-0,05$ & $-1,13$ & 0,45 & 4,26 & 3,67 \\
$48-57$ & 0,74 & $-0,54$ & $-1,25$ & $-0,48$ & $\underline{0,13}$ & $-1,85$ \\
$58-67$ & 8,27 & 6,85 & $\underline{-1,43}$ & $\underline{-1,39}$ & $-0,91$ & $-2,99$ \\
$68-77$ & 1,74 & $-0,36$ & $-7,48$ & $-7,59$ & $-5,77$ & $-9,21$ \\
$\geq 78$ & $\underline{3,62}$ & $\underline{4,91}$ & $-2,21$ & $-2,33$ & $-1,93$ & $-5,16$ \\
\hline
\end{tabular}

b) Nas idades técnicas de colheita (ITC), o modelo proposto apresentou-se menos preciso na menor e maior classe de produtividade.

\section{REFERÊNCIAS BIBLIOGRÁFICAS}

CAMPOS, J.C.C.; LEITE, H.G. Mensuração florestal. Viçosa, MG: UFV, 2002. 407p. 
CLUTTER, J.L. Compatible growth and yield models for lobolly pine. Forest Science, v.9, n.3, p.354-371, 1963.

CLUTTER, J.L. et al. Timber management: a quantitative approach. New York: John Wiley \& Sons, 1983. 333p.

DAVIS,L.S.; JOHNSON, K.N. Forest management. 3.ed. New York: McGraw-Hill, 1987.789p.
JONES, J.R. Review and comparison of site evaluation methods. Rocky Mountain For. Exp. Station, USDA: Forest Service, 1969. 27p. (Research Paper, RM-51)

LEARY, R. A. Some factors that will affect the next generation of forest growth models. In: FOREST Growth Modelling and Prediction Conference, 1., 1987, Minneapolis, Minnesota, USA:

Proceedings....Minneapolis: United States Departament of Agriculture, North Central Forest Experiment Station, v.1, p. 22-32, 1988. (General Technical Report NC - 120).

SPURR, S. Forest inventory. New York: Ronald Press, 1952.476p. 